\title{
The Entropy of Acetic Acid
}

\author{
J. O. HALFORD \\ Department of Chemistry, University of Michigan, Ann Arbor, Michigan
}

(Received August 8, 1941)

\begin{abstract}
From third law measurements, vapor pressures and vapor densities, the entropy of the acetic acid monomer at $25^{\circ}$ and one atmosphere is $69.4 \pm 1.0$ e.u. The value 68.7 is calculated from the vapor phase ethyl acetate equilibrium. For a model based upon acetone and approximately representing free rotation the entropy would be 72.7 . If there is only a single potential minimum in the hydroxyl group rotational cycle, the large deficiency below the free rotation value is explained without assuming an exceptionally high potential barrier. A brief discussion of the effect of the number of potential minima and their relative depth is appended, and a possible source of error in third law measurements is suggested.
\end{abstract}

$T^{1}$ $\mathrm{HE}$ entropy of the acetic acid monomer as a hypothetical perfect gas can be calculated from the third law entropy of the liquid and the heat of vaporization with the aid of the constants of the reaction

$$
\left(\mathrm{CH}_{3} \mathrm{COOH}\right)_{2}=2 \mathrm{CH}_{3} \mathrm{COOH} \text {. }
$$

This procedure or its equivalent is made necessary by the wide deviations of acetic acid vapor from perfect gas behavior. The significance and accuracy of the result depend upon the completeness with which existing vapor density data may be used to describe the vapor as a mixture of two perfect gases in equilibrium according to Eq. (1). Although it is reasonable to suppose that at low pressures this description should be valid, accurate vapor densities at any one temperature are not available over a wide enough range of pressures to establish the point by experiment. Consequently the entropy requires support from other sources. The calculation has therefore been made at a series of temperatures from 25 to $100^{\circ}$ and an average value of the heat capacity between these temperatures has been derived and shown to lie between the heat capacities of the related substances ethyl alcohol and acetone. As a further and more direct confirmation an independent value of the entropy is derived from the results of a recent study of the vapor phase ethyl acetate equilibrium.

In a similar study of the nitrogen dioxidenitrogen tetroxide case Giauque and Kemp ${ }^{2}$

1 The author is indebted to Dr. Donald Brundage for making preliminary calculations.

${ }^{2}$ W. F. Giauque and J. D. Kemp, J. Chem. Phys. 6, 41 (1938). concluded that the entropy of dissociation of the dimer could not be evaluated from equilibrium data within several entropy units. This uncertainty would of course be carried in some measure in to the entropy of each component. The acetic acid case appears to be more favorable.

Since the publication by MacDougall ${ }^{3}$ of vapor densities measured at low pressures near room temperature, it is possible to evaluate the heat of reaction 1 and the composition of the mixture as a function of temperature with a high probable accuracy: From MacDougall's results and those of Fenton and Garner, ${ }^{4}$ the heat of dissociation of the dimer becomes

$$
\Delta H=18,853-9.76 T
$$

and, for the dissociation constant,

$$
\log k=25.732-4120 / T-4.910 \log T \text {. }
$$

Constants calculated by Eq. (3) show an average deviation of 2.0 percent from MacDougall's mean values. If his point at $25^{\circ}$ is left out the deviation decreases to 0.8 percent. Similarly average constants from the Fenton and Garner data are re-

\begin{tabular}{|c|c|c|c|c|c|}
\hline $\begin{array}{l}\text { TEMP. } \\
\text { DEg. C }\end{array}$ & $\begin{array}{l}\Delta H \\
\text { Dis. }\end{array}$ & $\begin{array}{c}k \text { (DIS.) } \\
\mathbf{M M}\end{array}$ & $\stackrel{p}{M M}$ & $\alpha$ & $\begin{array}{l}\Delta H \\
\mathrm{VAP}\end{array}$ \\
\hline 25 & 15,944 & 0.578 & 15.63 & 0.0957 & 10,874 \\
\hline 40 & 15,797 & 2.080 & 34.72 & .1215 & 11,011 \\
\hline 60 & 15,602 & 9.48 & 88.96 & .1611 & 11,236 \\
\hline 80 & 15,407 & 35.8 & 202.4 & .2058 & 11,497 \\
\hline 100 & 15,212 & 115.1 & 417.2 & .2540 & 11,779 \\
\hline
\end{tabular}
produced with a mean deviation of 1.0 percent, or

TABLE I. Vaporization and association of acetic acid.

${ }^{3}$ MacDougall, J. Am. Chem. Soc. 58, 2585 (1936).

${ }^{4}$ Fenton and Garner, J. Chem. Soc. p. 694 (1930). 
TABLE II. Entropy of the acetic acid monomer.

\begin{tabular}{|c|c|c|c|c|c|c|}
\hline $\begin{array}{l}\text { TEMP. } \\
\text { DEG. }\end{array}$ & $\begin{array}{r}S(60 \\
\text { G LiQ.) }\end{array}$ & $\Delta H_{1}$ & $\Delta H_{1} / T$ & $p_{1}$ & $\begin{array}{c}R \text { LN } \\
760 / p_{1}\end{array}$ & $\begin{array}{c}S_{1} \\
\text { GAS }\end{array}$ \\
\hline 2.5 & $38.2^{0}$ & 12,646 & $42.4^{2}$ & $2.73^{1}$ & $11.2^{3}$ & 69.3 \\
\hline 40 & $39.6^{7}$ & 12,445 & $39.7^{5}$ & $7.52^{4}$ & $9.1^{7}$ & 70.2 \\
\hline 60 & $41.5^{9}$ & 12,162 & $36.5^{1}$ & $24.6^{9}$ & $6.8^{1}$ & 71.2 \\
\hline 80 & $43.4^{5}$ & 11,867 & $33.6^{1}$ & $69.0^{8}$ & $4.7^{7}$ & 72.2 \\
\hline 100 & $45.2^{8}$ & 11,564 & $30.9^{9}$ & 169.0 & $2.9^{9}$ & 73.2 \\
\hline
\end{tabular}

0.3 percent at three of their four temperatures. Results obtained by Drucker and Ullmann ${ }^{5}$ at three temperatures from 80 to $110^{\circ}$ are on the average 5 percent above the constants given by Eq. (3). If the weight at higher temperatures were given to the Drucker and Ullmann results it would be impossible to bring Eqs. (2) and (3) into line with the remaining data without an unreasonably large $\Delta C_{p}$ term expressed in greater detail. The heat of dissociation according to Eq. (2) is 15,944 cal. at $25^{\circ}$ as compared with $16,400 \pm 800$ obtained by MacDougall from his own data alone. Since it seems apparent that his equilibrium constant at $25^{\circ}$ is low, and his mean heat of reaction therefore high, the correct value should lie within the lower half of his range of uncertainty. Equation (2) gives heats of dissociation within these limits up to $60^{\circ}$.

The heat of vaporization is evaluated from the vapor pressures in the International Critical Tables, ${ }^{6}$ which are reproduced with an average deviation of 0.1 percent from 20 to $100^{\circ}$ by the equation

$$
\log p=18.8302-2630 / T-3.562 \log T
$$

For the vaporization of 120 grams of the acid

$$
\Delta H=(1+\alpha)(12035-7.08 T) .
$$

Table I contains the heat of dissociation, the degree of dissociation $\alpha$, the dissociation constant, the vapor pressure and the heat of vaporization calculated from the above equations at five temperatures. The heats of vaporization listed in the International Critical Tables ${ }^{7}$ and marked as questionable are as much as $320 \mathrm{cal}$. lower than those appearing in Table I. MacDougall's values, 11,000 at $25^{\circ}$ and 11,270 at $40^{\circ}$ are correspondingly high. They cannot be taken as more

${ }^{5}$ Drucker and Ullmann, Zeits. f. physik. Chemie 74, $567(1910)$.

${ }^{6}$ Vol. 3, p. 218

7 Vol. 5, p. 138 reliable, however, because they are based upon low pressures measured over a range of only $20^{\circ}$. If gas imperfections have been adequately accounted for in the factor $1+\alpha$, the heats of vaporization derived from Eq. (5) should not be in error by more than 100 calories.

The entropies of vaporization of the monomer and dimer are most readily obtained with the aid of individual heats of vaporization calculated from the equations

$$
\begin{aligned}
\Delta H_{1}(\text { vap. })=\Delta H(\text { vap. }) & / 2 \\
& +(1-\alpha) \Delta H(\text { dis. }) / 2
\end{aligned}
$$

and

$$
\Delta H_{2} \text { (vap.) }=\Delta H(\text { vap. })-\alpha \Delta H \text { (dis.) } .
$$

For 60 grams of liquid the entropy at $25^{\circ}$ is 38.2 according to Parks, Kelley and Huffman. ${ }^{8}$ The added liquid entropy for higher temperatures is calculated from the heat capacity given in the International Critical Tables. ${ }^{9}$ After conversion to calories this becomes $C_{p}=12.9+0.056 T$ in the appropriate temperature range. The results of the entropy calculation are shown in Table II for the monomer and in Table III for the dimer. The mean heat capacity of the monomer and dimer vapors is now derived from the entropy by means of the equation $d S / d T=C_{p} / T$. For the monomer, $C_{p}$ is $\mathbf{1 7 . 4}$ and for the dimer it is 44.1 . Because of structural similarity it is reasonable to assume that the monomer value should be near the mean of the heat capacities of ethyl alcohol and acetone. From Table IV these are, respectively, 16.8 and 19.9 at the mean temperature of $62^{\circ}$. For reaction $1, \Delta C_{p}$ is -9.3 as compared with -9.76 shown in Eq. (2). This discrepancy is probably due to an oversimplification of Eq. (5), for which the true heat capacity, there represented as $-7.08(1+\alpha)$, should have the more

TABLE III. Entropy of the acetic acid dimer.

\begin{tabular}{rccccc}
\hline $\begin{array}{c}\text { TEMP. } \\
\text { DEG. C }\end{array}$ & $\Delta H_{2}$ & $\Delta H_{2} / T$ & $p_{2}$ & $\begin{array}{c}R \text { LN } \\
760 / p_{2}\end{array}$ & $\begin{array}{c}S_{2} \\
\text { GAS }\end{array}$ \\
\hline 25 & 9349 & $31.3^{6}$ & 12.90 & $8.1^{0}$ & $99.6^{6}$ \\
40 & 9092 & $29.0^{4}$ & 27.20 & $6.6^{2}$ & $101.7^{6}$ \\
60 & 8722 & $26.1^{9}$ & 64.27 & $4.9^{1}$ & $104.4^{5}$ \\
80 & 8326 & $23.5^{8}$ & 133.3 & $3.4^{6}$ & $107.0^{3}$ \\
100 & 7916 & $21.2^{2}$ & 248.2 & $2.2^{2}$ & $109.5^{5}$ \\
\hline
\end{tabular}

${ }^{8}$ Parks, Kelley and Huffman. J. Am. Chem. Soc. 51, 1969 (1929).

${ }^{9}$ Vol. 5 , p. 114 
detailed form

$$
\begin{aligned}
\Delta C_{p}=2 \alpha C_{p 1}+ & (1-\alpha) C_{p 2} \\
& +\Delta H(\text { dis. }) d \alpha / d T-2 C_{p}(1) .
\end{aligned}
$$

Since the discrepancy involves only a fraction of an entropy unit, the second approximation required to produce consistency would not be justified by the data used.

The above calculation using individual heats of vaporization is different in form from the method outlined by Giauque and Kemp. ${ }^{2}$ Their approach leads to two equations, the one expressing the two unknowns in terms of the entropy of dissociation of the dimer, the other relating them to the entropy of the mixture and the entropy of mixing. The two methods can readily be shown to be equivalent, either by algebraic proof or by numerical substitution.

If the entropy calculation is made from MacDougall's ${ }^{3}$ data alone the results for $S_{1}$ and $S_{2}$ at $25^{\circ}$ are 70.3 and 100.0 , which represent practical maximum values and show that the entropies are not highly sensitive to the interpretation of the data.

A further estimate of the monomer entropy can be made from the result of a recent study of the vapor phase ethyl acetate equilibrium by Halford and Brundage. ${ }^{10}$ For the reaction

$$
\begin{aligned}
\mathrm{CH}_{3} \mathrm{COOH}(\mathrm{g}) & +\mathrm{C}_{2} \mathrm{H}_{5} \mathrm{OH}(\mathrm{g}) \\
& =\mathrm{CH}_{3} \mathrm{COOC}{ }_{2} \mathrm{H}_{5}(g)+\mathrm{H}_{2} \mathrm{O}(\mathrm{g}),
\end{aligned}
$$

the minimum mean value of the entropy reasonably derivable from existing equilibrium data between 40 and $230^{\circ}$ is -3.3 units. To derive the value at $25^{\circ}$ requires knowledge of the heat capacities of the reactants. Table IV shows estimated values together with references to the data upon which they are based. In the absence of direct data, acetic acid is assumed to have the

TABLE IV. Heat capacity of gases, 25 to $150^{\circ}, C_{p}=A+B T$.

\begin{tabular}{lccc}
\hline & $A$ & $B$ & REFERENCES \\
\hline $\mathrm{H}_{2} \mathrm{O}$ & 7.47 & 0.0017 & (a) \\
$\mathrm{C}_{2} \mathrm{H}_{5} \mathrm{OH}$ & 3.4 & .040 & (b) \\
$\mathrm{CH}_{3} \mathrm{COOC}{ }_{2} \mathrm{H}_{5}$ & 8.9 & .060 & (b), (c) \\
$\mathrm{CH}_{3} \mathrm{COOH}_{\mathrm{CH}_{3} \mathrm{COCH}_{3}}$ & 5.1 & .037 & Estimate \\
& 8.5 & .034 & (b), (c)
\end{tabular}

a A. R. Gordon, J. Chem. Phys. 2, 65 (1934)

bennewitz and Rossner, Zeits. f. physik. Chemie B39, 134 (1938). International Critical Tables, Vol 5, p. 79

\begin{tabular}{|c|c|c|c|c|}
\hline & $\begin{array}{c}S \\
\text { Lia. }\end{array}$ & $\begin{array}{l}\Delta H \\
\text { VAP. }\end{array}$ & $\begin{array}{c}p \\
\text { мM }\end{array}$ & $\underset{\text { GAS }}{S}$ \\
\hline $\begin{array}{l}\text { Ethyl Alcohol } \\
\text { Ethyl Acetate }\end{array}$ & $\begin{array}{l}38.4 \\
62.0\end{array}$ & $\begin{array}{r}10,125 \\
8,596\end{array}$ & 58.6 & $\begin{array}{l}67.3 \\
86.7\end{array}$ \\
\hline Acetone & 47.8 & 7,488 & 229.2 & 70.6 \\
\hline
\end{tabular}

${ }^{10} \mathrm{~J}$. O. Halford and D. Brundage, in press.
TABLE V. Entropy of ethyl alcohol, ethyl acetate and acetone at $25^{\circ}$.

mean temperature dependence of ethyl alcohol and acetone and the constant term is set to give the value 17.4 at $62^{\circ}$ consistent with the entropy calculation. The experimental entropy of reaction 9 is assumed to be correct at the mean temperature of $135^{\circ}$ and the entropy of esterification is calculated with the aid of the above heat capacities to be -4.2 at $25^{\circ}$. There is no good basis for estimating the probable error of this value.

The entropy of water vapor at $25^{\circ}$ and one atmosphere is taken as $45.100^{11,12}$ For ethyl alcohol, ethyl acetate and acetone the required quantities are summarized in Table V. In the order given the liquid entropies are those of Kelley, ${ }^{13}$ Parks, Huffman and Barmore, ${ }^{14}$ and Parks, Kelley and Huffman. ${ }^{8}$ Both the vapor pressure and the heat of vaporization of the alcohol are values published by Fiock, Ginnings and Holton. ${ }^{15}$ Other vapor pressures come from the International Critical Tables ${ }^{6}$ and the heats of vaporization are taken from the vapor pressure curves and corrected by means of the equation $P V=R T(1-a P)$, for which $a$ is evaluated from the Mathews ${ }^{16}$ heats of vaporization at the respective boiling points. The similarly derived value for the alcohol is 10,150 or 10,195 cal., according to whether the Fiock, Ginnings and Holton heat of vaporization or that of Mathews is taken as correct at the boiling point.

From the entropy of esterification and the results in Table $\mathrm{V}$ the entropy of acetic acid at $25^{\circ}$ and one atmosphere is estimated to be 68.7 . Although this estimate in itself is subject to considerable error, it nevertheless contributes

11 A. R. Gordon, J. Chem. Phys. 2, 65 (1934).

${ }_{12}$ Giauque and Stout, J. Am. Chem. Soc. 58, 1144 (1936); Giauque and Archibald, J. Am. Chem. Soc. 59, 561 (1937).

${ }_{13}$ Kelley, J. Am. Chem. Soc. 51, 779 (1929).

14 Parks, Huffman and Barmore, J. Am. Chem. Soc. 55, 2733 (1933).

${ }^{15}$ Fiock, Ginnings and Holtón, J. Research Nat. Bur. Stand. 6, 892 (1931).

${ }_{16}$ Mathews, J. Am. Chem. Soc. 48, 572 (1926). 
TABLE VI. Acetic acid entropy from the entropy of acetone.

\begin{tabular}{lcc} 
& & \\
\hline & ACETONE & ACETK ACID \\
\hline$M$ & 58.08 & 60.05 \\
$A B C$ & 1.48 & $1.20,1.23$ \\
$o$ & 18 & 3 \\
$I_{\mathrm{CH}_{3}}$ & 0.0527 & 0.0527 \\
$I_{\mathrm{OH}}$ & - & 0.0131 \\
$S(\mathrm{vib})$. & 2.4 & 2.4 \\
$S_{298}$ & 70.6 & 72.7 \\
\hline
\end{tabular}

strongly to the high probability already established that the entropy of the acid monomer lies within the limits $69.4 \pm 1.0$.

For comparison, a theoretical value is calculated from the entropy of acetone with the aid of the equation

$S_{298}=23.730+(3 / 2) R \ln M+8.712 n$

$+(1 / 2) R \ln \left(A^{a} B^{b} \ldots G^{g}\right)-R \ln \sigma+S$ (vib.)

in which $M$ is the molecular weight in grams, $A$, $B$, etc., are moments of inertia, external and internal, in c.g.s. units times $10^{38}, a+b+\cdots+g=n$ is the number of rotational degrees of freedom and $\sigma$ is the symmetry number. Equation (10), for the entropy with free internal rotation, differs only negligibly from the theoretically correct form derived by Kassel. ${ }^{17}$ It is assumed in its application to acetic acid that the hydroxyl group may be described closely enough as a symmetrical internal rotator. The vibrational entropy of acetone is derived from the total entropy in Table $\mathrm{V}$ by the use of the above equation, and should therefore be low by an amount representing the restriction of internal rotation. For acetone the restricting barrier is probably small, ${ }^{18}$ corresponding almost to free rotation. Acetic acid is then assumed to have the same vibrational entropy, and its total entropy, shown in Table VI, should therefore be only a little lower than the free rotation value.

This approach, rather than the customary one involving a questionable independent estimate of the vibrational contribution, is of special interest because it can be used to calculate the entropy of methyl alcohol from that of ethane or of ethyl alcohol from propane within a small fraction of an entropy unit. Consequently the result of

${ }^{17}$ L. S. Kassel, J. Chem. Phys. 4, 276 (1936).

${ }^{18}$ S. C. Schumann and J. G. Aston, J. Chem. Phys. 6, 485 (1938).
Table VI is the entropy which acetic acid would have if the substitution of the hydroxyl group for a methyl group produced the same changes in all three molecules, ethane, propane and acetone.

In calculating the external moment product $A B C$ and the moments of inertia $I$ of the internal rotators, all angles between single bonds were assigned the tetrahedral value, and interatomic distances were taken as $c-c=1.54 \mathrm{~A}, c-h=1.09$, $c-o=1.43, c=o=1.24$ and $o-h=0.97$. For acetic acid the two tabulated moment products were calculated for models with the acid hydrogen in the cis-and trans-positions relative to the carbonyl oxygen. The hydroxyl group was assumed to rotate as if its center of gravity were on the $\mathrm{C}-\mathrm{O}$ bond.

It appears from the above results that restricted rotation of the acid hydroxyl group causes an entropy decrease below the free rotation value which exceeds the corresponding effect in methyl or ethyl alcohol by nearly three units. This large deficiency can be readily explained in a way that indicates that the experimental entropy of 69.4 is not far from correct. It is reasonable to assume that in the entire rotational cycle there is only a single potential minimum of sufficient depth to trap the hydroxyl group. For such a model, with vibration restricted to the ground state, the entropy would be 68.3 plus a small increment entering through the use of the acetone vibrational entropy. The experimental value thus allows 1.1 e.u. to be divided between this increment and the actual entropy of the torsional motion.

Although Pitzer's ${ }^{19}$ method of finding the restricting potential barrier was developed for $n$ equal sinusoidal potential valleys each covering one $n$th of the cycle, his table can be used for the single minimum model postulated for acetic acid. The case is similar to his treatment of the individual forms of $n$-butane. ${ }^{20} \mathrm{It}$ is assumed that the single minimum occupies one-third of the cycle. For an imaginary model with two more minima equal in depth to the first the entropy would be 69.4 plus a mixing term $R \ln 3$, giving $S_{f}-S=1.1$ for use in the table. The resulting potential barrier is about 3000 cal., a value which

${ }^{19} \mathrm{~K}$. S. Pitzer, J. Chem. Phys. 5, $469(1937)$.
${ }_{20} \mathrm{~K}$. S. Pitzer, J. Chem. Phys. 5, $473(1937)$. 
is subject to an error, probably negligible, because the table applies only when the rotational states are symmetrically disturbed in $n$ regions of the cycle, rather than unsymmetrically as they would be in the single minimum case.

In general, for the hydroxyl group in torsional motion against an unsymmetrical radical, it will be necessary to add a mixing term to the experimental entropy before a potential barrier can be estimated by means of Pitzer's table or the equivalent functions. This term has a maximum value $R \ln n$, but may be near zero if the potential valleys approach equality in depth. With ethyl alcohol, for example, there are probably three minima of which two are equal but different from the third, and a mixing term between zero and
$R \ln 3$ should be added to the experimental entropy before the barrier is estimated. If the correction is not made the barrier will be high.

An interesting possibility occurs for substances which have stable torsional vibrations resulting in optically isomeric configurations. The molecules might remain asymmetric after crystallization and might produce crystals of the racemic compound type which would remain unchanged upon cooling toward the absolute zero. Consequently the entropy at the absolute zero would not be zero but $R \ln 2$, and the third law entropy would be in error by this amount. How probable this situation is depends upon the relative magnitude of intramolecular and intermolecular forces in the solid state.

\title{
Second Virial Coefficients of Polar Gas Mixtures $\ddagger$
}

\author{
W. H. StockmayeR* \\ Research Laboratory of Physical Chemistry, Massachusetts Institute of Technology, Cambridge, Massachusetts
}

(Received October 15, 1941)

\begin{abstract}
An empirical equation used by Keyes to represent the second virial coefficients of several polar gases is compared to a theoretical expression for the same quantity. The constants appearing in this equation are discussed in terms of intermolecular forces, and their relationship to analogous constants in the Beattie-Bridgeman equation for non-polar gases is examined. These considerations permit the construction of simple rules by means of which the second virial coefficient of any gas mixture may be calculated if the second virial coefficients and dipole moments of the pure component gases are known. The success of these rules is demonstrated by comparison with data on $\mathrm{H}_{2} \mathrm{O}-\mathrm{CO}_{2}, \mathrm{~N}_{2}-\mathrm{NH}_{3}$, and $\mathrm{N}_{2}-\mathrm{H}_{2} \mathrm{O}$ systems. In the case of $\mathrm{N}_{2}-\mathrm{H}_{2} \mathrm{O}$ mixtures (vapor-liquid equilibrium data) the agreement is not satisfactory at the higher temperatures. A rough method of including the effect of higher virial coefficients reduces, but does not remove, this discrepancy.
\end{abstract}

\section{INTRODUCTION}

$\mathrm{T}$ HE Beattie-Bridgeman equation of state for gas mixtures ${ }^{1}$ has been applied successfully not only to the calculation of the compressibility of many gas mixtures, but also to the correlation of several types of high pressure equilibrium data. ${ }^{2}$ A substance conspicuously

$\ddagger$ Contribution from the Research Laboratory of Physical Chemistry, Massachusetts Institute of Technology, No. 483. * Present address, Department of Chemistry, Columbia University, New York.

I J. A. Beattie, J. Am. Chem. Soc. 51, 19 (1929); J. A. Beattie and S. Ikehara, Proc. Am. Acad. 64, 127 (1930).

${ }^{2}$ L. J. Gillespie, Chem. Rev. 18, 359 (1936), where a complete list of references to important earlier work is absent from all these calculations is steam, for the reason that the experimental $p-V-T$ data $^{3}$ cannot be adequately represented by an equation of the Beattie-Bridgeman form. The curvature of the isometric lines for steam is so great that the ordinary Beattie-Bridgeman function is unsatisfactory. Theoretically this great curvature may be shown to be a direct consequence of the nature of the forces between highly polar mole-

\footnotetext{
given. See especially Gillespie, J. Am. Chem. Soc. 47, 305 and 3106 (1925); Gillespie and Beattie, Phys. Rev. 36, 743, 1008 (1930).

${ }^{3}$ F. G. Keyes, L. B. Smith, and H. T. Gerry, Proc. Am. Acad. 70, 319 (1936).
} 